\title{
La adquisición de segmentos del inglés desde la sociolingüística: la fricativa postalveolar sorda $/ \int /^{*}$
}

\author{
The Acquisition of English Segments from a Sociolinguistic \\ Perspective: The Voiceless Postalveolar Fricative $/ \mathrm{J} /$
}

Paulina B. Subiabre Ubilla **

\section{RESUMEN}

En la tradición laboviana el estudio de la variación lingüística Palabras clave: ha comenzado a aplicarse recientemente en investigaciones de variación segunda lengua, donde se ha demostrado que en este tipo de contextos (principalmente inmigración) surgen patrones sociolingüísticos similares a los encontrados en estudios de variación en lengua materna. Este artículo recoge la fricativa postalveolar sorda $\left(\int\right)^{* * *}$ como variable fonética en el habla de 18 estudiantes chilenos de inglés. Por medio de análisis de factor por factor y lingüística, fonética acústica, transferencia, hipercorrección, inglés como lengua extranjera. de variable múltiple se buscó determinar, en primer lugar, si los patrones de variación lingüística del español de Chile también ocurren en situaciones de inglés como lengua extranjera, dado que esta variable ha sido históricamente correlacionada con factores sociales y, en segundo término, si los hablantes no nativos de inglés replican la variación que muestran los hablantes nativos. Los resultados sugieren que tanto factores lingüísticos como sociales contribuyen a la variación de ( $($ ). De esta forma, el artículo contribuye a fortalecer la noción de que la variación es inherente a las lenguas, tanto en lengua materna como en lengua extranjera.

La variable estudiada en este artículo corresponde a uno de los capítulos de la tesis doctoral "A phonetic variationist study on Chilean speakers of English as a foreign language", de mi autoría.

** Chilena, $\mathrm{PhD}$ in English Language, University of Glasgow, académica Universidad del Bío-Bío, Chillán, Chile. psubiabre@ubiobio.cl

*** En sociolingüística los paréntesis se utilizan para referirse a una variable lingüística; las variantes lingüísticas (las distintas realizaciones de una variable) serán identificadas con corchetes. 


\begin{abstract}
Variationist research in the Labovian paradigm has traditionally looked at the structured heterogeneity of first languages. This methodology has been recently applied to second languages, usually in immigration settings. Research has shown sociolinguistic patterns in the $\mathrm{L} 2$ acquisition processes that are similar to those found in $\mathrm{L} 1$ speech. This article examines the speech of 18 Chilean university learners of English and explores the variation of voiceless postalveolar fricative ( $($ )). Using a factor by factor and multivariate analysis, it seeks to first test whether the patterns of variation characteristic of Chilean Spanish are transferred to English; and secondly, whether the variation exhibited by native speakers of English is replicated in EFL contexts. The results suggest that the transfer of patterns from the L1 to the L2 occurs for ( () ). Both social and linguistic factors have an effect on the use of $(\delta)$. The study of variation in EFL seeks to contribute to the notion that variation is inherent to language in native and non-native contexts, especially in relation to the effect of internal constraints.
\end{abstract}

Keywords: linguistic variation, acoustic phonetics, transfer, overcorrection, English as a foreign language. 


\section{Introducción}

El estudio cuantitativo de la variación lingüística se basa en el argumento de que todas las lenguas naturales varían y cambian (Sapir, 1921; Weinreich, Lavov \& Herzog, 1968) y, dentro de ella, la perspectiva laboviana sugiere que dicha variación es el resultado de la convergencia de factores lingüísticos y extralingüísticos. Tal variación es inherente a la lengua y tiende a ser sistemática, por lo que se espera que todos los hablantes de un idioma varíen sistemáticamente en un rango de situaciones lingüísticas, dependiendo de factores internos (lingüísticos) y/o externos (sociales). Dentro de los factores internos que han presentado históricamente una mayor incidencia en la variación lingüística se cuentan el contexto fonético inmediato y la posición de la variable. Asimismo, los factores externos más recurrentes son la edad, el sexo, el nivel educacional y el nivel socioeconómico de los hablantes.

Si bien el estudio de la variación lingüística se ha enfocado tradicionalmente en la variación en lengua materna (L1), las últimas décadas han visto un aumento en el estudio de la variación de hablantes de segunda lengua (L2), principalmente en contextos de inmigración y aún más recientemente, en casos de lengua extranjera (por ejemplo, Kpogo \& Gathercole, 2019; Miatto, Hamann \& Boersma, 2019), donde el habla que se adquiere no ocurre como componente de un proceso de inmersión, como en el caso de Chile. El español de Chile presenta ciertas características dialectales que lo diferencian de otras variedades de español, entre las que se cuentan el uso de la fricativa postalveolar sorda que se analiza acá. El presente estudio buscó determinar si la variación fonética que exhiben los hablantes de inglés como lengua extranjera (ILE) es sistemática y, de ser así, si esta variación es atribuible a factores sociales, lingüísticos o a ambos; $\mathrm{y}$, en segundo término, comprobar si la variación en hablantes de ILE es similar a la de los hablantes nativos, al menos en lo que respecta a esta variable.

El artículo examina el uso de la fricativa postalveolar sorda en el habla de 18 alumnos de la carrera de Pedagogía en Inglés de la Universidad del Biobío, Chillán, en relación con los factores que se muestran en la Tabla 1. 
Tabla 1.

Factores externos e internos considerados en el análisis de la variación de la fricativa postalveolar sorda.

\begin{tabular}{|c|c|c|}
\hline Tipo de factor & Grupo de factores & Factores \\
\hline \multirow{8}{*}{$\begin{array}{l}\text { Externo } \\
\text { (social) }\end{array}$} & \multirow[t]{2}{*}{ Sexo } & Hombre (M) \\
\hline & & Mujer (F) \\
\hline & \multirow{3}{*}{$\begin{array}{l}\text { Exposición al inglés en } \\
\text { contexto educativo (en años) }\end{array}$} & 1 \\
\hline & & 3 \\
\hline & & 5 \\
\hline & \multirow[t]{3}{*}{ Grupo socioeconómico } & $\mathrm{C} 2$ \\
\hline & & $\mathrm{C} 3$ \\
\hline & & $\mathrm{D}$ \\
\hline \multirow{7}{*}{$\begin{array}{l}\text { Interno } \\
\text { (lingüístico) }\end{array}$} & \multirow{3}{*}{$\begin{array}{l}\text { Contexto fonético (anterior y } \\
\text { posterior) }\end{array}$} & Vocales \\
\hline & & Consonantes \\
\hline & & Pausa \\
\hline & \multirow[t]{2}{*}{ Posición } & Inicio de turno \\
\hline & & $\begin{array}{l}\text { Otra posición en el turno: } \\
\text { Inicial (en la palabra) } \\
\text { Interna (en la palabra) } \\
\text { Final (en la palabra) }\end{array}$ \\
\hline & \multirow[t]{2}{*}{ Clase de palabra } & Abierta \\
\hline & & Cerrada \\
\hline
\end{tabular}

Fuente: Elaboración propia.

\section{Marco teórico}

La fricativa postalveolar sorda $(\delta)$ aparece en una variedad de contextos lingüísticos en inglés, como en los lexemas show $/$ Jəo/ $/{ }^{1}$, perception

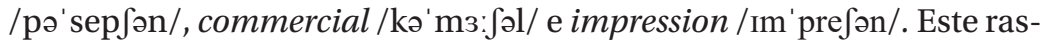
go no ha sido estudiado como variable en hablantes nativos de inglés, pero sí aparece en descripciones fonéticas generales del inglés (Catford, 2002; Ladefoged \& Disner, 2012) y asociado al efecto que tienen las vocales en su realización (Shadle, Proctor, \& Iskarous, 2008; Wilde, 1995). También ha sido mencionado como variante de (s) en el habla de

$1 \quad$ Esta y las siguientes transcripciones fonéticas estándares corresponden al acento RP (received pronunciation), uno de los más tradicionales en la enseñanza del inglés como lengua extranjera. 
Glasgow (Stuart-Smith, 2007; Stuart-Smith, Timmins \& Wrench, 2003), en relación con la percepción de identidad sexual (Levon, 2007) y en la alternancia de $(\mathrm{sh} / \mathrm{ch})^{2}$ en hablantes latinos en Nueva York (Labov, 2012). En español, el rasgo ha sido estudiado como una de las variantes para la africada alveolar sorda $(\mathfrak{t})$ ), asociada principalmente a factores externos como género y clase social (Cedergren, 1973, 1987; Valencia, 1993-1994), con una variación que va desde una variante prestigiosa en Panamá hasta la estigmatizada en Chile.

\subsection{Variación en inglés}

La variación de $(\delta)$ se ha descrito principalmente desde su articulación, con un enfoque bastante limitado en la lingüística variacionista (Catford, 2002; Jongman, Wayland \& Wong, 2000; Ladefoged \& Disner, 2012; Ladefoged \& Johnson, 2011; Li, Edwards \& Beckman, 2009). Dentro de esta, la variable ha sido estudiada solo como una variante de (s) asociada con la clase social de los hablantes de Glasgow (Smith, 2007; Stuart-Smith et al., 2003) o con la identidad sexual (Levon, 2007). Sin duda, es un elemento que presenta variación en inglés, pero el valor sociolingüístico de (sh) en el español de Chile es de naturaleza distinta.

\subsection{Variación en español}

La fricativa postalveolar sorda - a diferencia del inglés-, no tiene una correspondencia ortográfica directa en ninguna de las variedades de español; es más, no es considerada en el inventario estándar del español, apareciendo como un alófono en variación libre y posiblemente en distribución complementaria con (t f) (Dalbor, 1959). En el inventario no estándar ocurre en posición intervocálica en palabras como muchacho (estándar [mutfatfo] versus no estándar [mu' $\mathrm{a} \mathrm{a} \mathrm{o}$ ]), después de consonantes en ancho (estándar ['antfo] versus no estándar ['anfo]) y en posición inicial en chala (estándar ['ţala] versus no estándar ['Jala]); en español, este elemento no ocurre en posición final, salvo en el caso de préstamos.

Labov (2012) utiliza la notación (sh/ch) para el estudio de las representaciones grafémicas más comunes para la fricativa alveolar sorda $/ \mathrm{J} /(\mathrm{sh})$ y la africada alveolar sorda $/ \mathrm{t} /$ / (ch). 
Como variable ( () ha sido documentada en variedades de la República Dominicana, Cuba, en la región española de Andalucía y Nuevo México (Canfield, 1962; Cedergren, 1973; Lipski, 1994). En Ciudad de Panamá, Cedergren $(1973,1987)$ examinó la lenición de $<\mathrm{ch}>$, definida como la "alternancia de una palatal fricativa [̌̌ con la estándar [č]"3 (Cedergren, 1987, p. 51); los factores considerados en su estudio fueron la posición prosódica en la palabra (inicial y media), el contexto fonológico, el estilo, el lugar de origen de los hablantes, el sexo, la edad y el grupo socioeconómico. Cedergren (1973) planteó que existían tres variantes en el español de Ciudad de Panamá: una africada [t] , una africada palatal sorda con un onset oclusivo reducido $[\mathrm{t} f]^{4}$, y una fricativa palatal sorda no labializada $\left[\int\right]^{5}$. Los resultados de su investigación -la diseminación del cambio fonético desde [tg] a [j], desde Ciudad de Panamá a otras ciudades- se comprobaron media generación más tarde (Cedergren, 1987), siendo el factor de mayor incidencia la edad de los hablantes (adultos jóvenes), que Cedergren interpretó como un indicador de la sensibilidad de este grupo etario a la importancia de la nueva variable, comparable con los patrones de hipercorrección descubiertos por Labov (1966), en Nueva York.

Las conclusiones de la autora también sugieren que la variación presenta un uso consistente de la variante prestigiosa en términos de sexo y que la reducción de la oclusión es favorecida cuando la preceden vocales y pausas. La presencia de un patrón curvilíneo en la variación de (ch) a partir de factores socioeconómicos mostró que los grupos opuestos favorecían $[\mathrm{t} f]$ y $[\mathrm{J}]$. De esta forma, Cedergren demostró que la variación de $(J)$ ocurre como resultado de la interacción de factores sociales y lingüísticos, específicamente edad, sexo y lugar de origen y segmento fonético anterior y posición prosódica. Sin duda, este trabajo sentó las bases para el estudio de ( $(S)$ en español, al confirmar que el uso de esta variable estaba condicionado por factores internos y externos en hablantes nativos de español.

\footnotetext{
3 Los fonos americanistas [š] y [č corresponden a los signos del alfabeto fonético internacional IPA [f] y $[\mathrm{t}]$, respectivamente.

Mi propia notación; Cedergren (1973) utiliza s.

Cedergren (1973) concluyó que el uso de la fricativa alveolar sorda es un cambio lingüístico de aparición reciente que se origina en las áreas urbanas y que fue iniciada por adultos jóvenes de clase media. Este proceso estuvo marcado por la implementación gradual fonética $[\mathrm{t}]>[\mathrm{t} f]>[\mathrm{S}]$.
} 
Sin embargo, los valores asignados a esta variable en Ciudad de Panamá se oponen a los estudiados en el español de Chile: en este contexto, el uso de la fricativa postalveolar sorda está determinado por factores sociales como la edad y el lugar de origen, pero el principal factor es la clase social de los hablantes (Tassara, 1992; Valdivieso, 1983). Al respecto, Bernales (1978) determinó que el nivel educacional, el sexo y el estilo (casual versus enfático) y la posición (inicial, intervocálica y postconsonántica) son todos factores que contribuyen a que (tg) sea producida como [ $]$.

Por último, Widgorsky (1978), Valencia (1993-1994) y Cepeda (2001) han demostrado que este sonido es característico de individuos de clase social baja, mientras que Valdivieso (1993) sugirió que los hablantes hombres de clase social baja muestran tasas más altas de uso de la variante no estándar, en este caso ( ()), que las hablantes de clase alta.

\subsection{Variación en contextos de inglés como lengua extranjera o segunda lengua}

El estudio de este fonema en contextos en que el inglés no es la primera lengua de sus hablantes, revela la aparición de al menos dos variantes que coinciden con las variantes encontradas en el español de Chile: la fricativa $\left[\int\right]$ y la africada [t] $]$. Así, al estudiar a un hablante cubano que aprendía inglés en Estados Unidos, Dalbor $(1959)^{6}$ determinó que la variación de estos sonidos resultaba de la dificultad de los hablantes para reconocer las distinciones fonéticas entre dichos sonidos y que este error podría corregirse con un entrenamiento apropiado. Por su parte, Eklund y Lindström (1998) sugirieron que la inclusión de xenófonos - como el caso de la fricativa alveolar sorda- está también presente en hablantes de sueco y Flege (1991) detectó, al estudiar a hablantes nativos de inglés que aprendían español con hablantes nativos de español, que estos producían la fricativa $(\delta)$ como $[\check{c}]^{7}$, lo que sugiere que la variación de este sonido se asocia al español en general. Finalmente, Labov (2012) estudió la alternancia de (ch/sh) en pasajes

\footnotetext{
${ }^{6} \quad$ El estudio de Dalbor no es variacionista, pero se basa en la observación del proceso adquisitivo desde una perspectiva educacional en contextos de inglés como segunda lengua o como lengua extranjera (EFL/ESL), donde la variación se considera como utilización errónea de ciertos elementos.

$7 \quad$ Ver la nota a pie $n^{\circ} 5$ del presente artículo.
} 
de lectura en hablantes de tres orígenes étnicos diferentes de Nueva York y Filadelfia: afroamericanos, caucásicos y latinos; dentro de este último grupo, se distinguieron los latinos que habían aprendido a leer primero en español de los que habían aprendido a hacerlo primero en inglés. Para Labov, la ocurrencia de "errores" es diferente para afroamericanos y latinos, ya que los errores del primer grupo son gramaticales y los del segundo, gramaticales y fonológicos. La conclusión de Labov es que esta alternancia entre la africada $/ \mathrm{t} / \mathrm{y}$ la fricativa $/ \mathrm{J} /$ ocurriría como consecuencia del aprendizaje de la lectura en español en primera instancia, pero los errores no tendrían consecuencias directas en la lectura o comprensión de textos en inglés.

\subsection{Hipótesis}

Dado el estatus de este sonido en el español de Chile (un estereotipo característico principalmente de hablantes de clase social baja, no educados), este trabajo propone que la variación sistemática de (J) que se evidencia en hablantes nativos de español, se replica en el habla del inglés como lengua extranjera, por medio de la trasferencia de la variante prestigiosa, como producto del fenómeno de hipercorrección ${ }^{8}$. Específicamente, y basada en estudios similares, la investigación buscó probar que:

- existe una diferencia entre el habla de hombres y mujeres no nativos de inglés, donde hay dos escenarios posibles: las mujeres muestran un mayor uso de la forma no estándar del inglés [tg] sobre la estándar [J], que es estigmatizada en el español de Chile, o bien, utilizan la forma estándar por sobre la no estándar (refiérase a la paradoja de género de Labov, 2001).

- el efecto del contexto fonético inmediato es uno de los factores más importantes que contribuyen a la variación, especialmente en presencia de vocales y pausa, ya que estas favorecen el proceso de lenición. 
- el tiempo de instrucción formal en inglés como lengua extranjera es también un factor importante, en particular en lo que respecta a la adquisición y producción de las formas meta.

- la lenición es más común en ítems léxicos de clase cerrada, en oposición a la fortición en ítems léxicos de clase abierta.

- hay un mayor uso de las variantes más débiles en posiciones media y final y de las variantes más fuertes en posición inicial.

\section{Metodología}

La metodología cuantitativa tradicional sugiere que una de las herramientas más eficientes en el estudio de la variación lingüística es la entrevista sociolingüística. Esta tiene tanto ventajas como desventajas: si bien permite que el investigador monitoree de forma confiable al objeto de estudio (en lo que respecta a la calidad de los materiales, grabación, etc.) y en cuanto a la obtención de datos demográficos ${ }^{9}$, aun así es una entrevista y el registro tiende a ser un poco más formal que si se tratara de una conversación entre conocidos (refiérase a la paradoja del observador, Labov, 1972).

Se obtuvieron más de 17 horas de datos, producto de entrevistas semiestructuradas a 18 estudiantes de la carrera de Pedagogía en Inglés de la Universidad del Biobío. La selección del número de estudiantes buscó garantizar la representatividad de la muestra en términos de sexo, formación y grupos socioeconómico (tres hombres y tres mujeres de primer, segundo y tercer año; la indexación del grupo socioeconómico fue posterior a la obtención de datos demográficos). Las entrevistas semiestructuradas se realizaron sobre la base de módulos de conversaciones (Labov, 1984).

Las conversaciones fueron grabadas y posteriormente transcritas grafémicamente en CLAN (MacWhinney, $(2000)^{10}$. Una vez finalizado el proceso de transcripción - que se estandarizó por medio de un protocolo de transcripción-, se identificaron las variables seleccionadas,

\footnotetext{
9 En oposición, por ejemplo, a la metodología empleada por Labov (1966a) en el estudio de las tiendas de departamentos en New York, donde el investigador se basó en características demográficas evidentes y no entrevistó directamente a los sujetos para la obtención de los datos demográficos.

10 CLAN, https://talkbank.org/software/
} 
al igual que el contexto fonológico inmediato y se codificaron los factores mencionados anteriormente. El análisis estadístico se realizó en el software GoldVarb (Tagliamonte, 2006), que permite estudiar de forma simultánea las variables lingüísticas por medio de análisis de factor por factor y luego de variable múltiple ${ }^{11}$.

\section{Resultados}

\subsection{Variantes y distribución}

Inicialmente se identificaron cuatro variantes:

- Fricativa postalveolar sorda [J], que correspondería al sonido meta del inglés y es estigmatizada en español de Chile. Se le denominará "fricativa" 12 .

- Africada postalveolar sorda [t ], forma no meta que corresponde a la variante de prestigio del español de Chile. Se denominará "africada".

- Oclusiva alveolar sorda [t], forma no meta, que no ha sido registrada previamente en estudios similares; se le denominará "oclusiva".

- Una forma de africada alveolar sorda con énfasis en la fricación [t]]; forma no meta, que se denominará "fricativizada". Esta variante fue registrada por Cedergren $(1973)^{13}$ y constituye una forma compuesta ${ }^{14}$ en que la africada y la fricativa comparten, al menos, uno de sus constituyentes (Chambers \& Trudgill, 1998). De acuerdo con Chambers (1980), estas formas son comunes en situaciones de contacto lingüístico entre lenguas o dialectos; en el caso de esta investigación [t $\mathrm{f}]$ surge del contacto entre el español (L1) y el inglés (L2).

A pesar de la cantidad limitada de estudios de la fricativa alveolar sorda $(\delta)$ en hablantes nativos de inglés, esta investigación se enfocó en determinar la fuente y los patrones de variación en hablantes no nativos, ya que el rasgo en estudio ha demostrado variación sistemática

\footnotetext{
11 Todos los aspectos metodológicos están detallados en Subiabre (2015).

12 La denominación de las variantes obedece a un aspecto práctico.

13 Cedergren (1973) la describe como una forma de africada palatal sorda con un onset oclusivo reducido. Esta forma ocurre en el $8 \%$ de las instancias totales de su estudio.

14 Chambers y Trudgill (1998) utilizan en término "fudge".
} 
por efecto de factores internos y externos. Específicamente, se buscó determinar si los mismos patrones descubiertos por Valencia (1983), Valdivieso (1983), Tassara (1992) y Cedergren (1973) ${ }^{15}$ en hablantes de L1 surgen en contextos de inglés como lengua extranjera.

Los resultados preliminares sugieren que nueve de los 18 hablantes estudiados no transfieren los patrones sociolingüísticos que se evidencian en español de Chile; en otras palabras, el $50 \%$ de la muestra utiliza la forma meta / $/$ / en lugar de evitarla, a pesar de ser una forma estigmatizada en su Ll. Es más, aunque este sonido no forma parte del inventario fonético del español estándar, se evidencia que su uso es aparentemente correlativo con la experiencia de los hablantes en la lengua extranjera: gran parte de los hablantes de quinto año son categóricos en su uso, seguidos de los hablantes de tercer año.

Siguiendo el tratamiento de hablantes categóricos de Tagliamonte (2006), solo se estudiaron aquellos individuos que mostraban variación por dos motivos: primero, los hablantes categóricos no varían, por lo que no es posible determinar el origen de la variación y segundo, la incorporación de estos individuos alteraría los resultados de los hablantes que sí varían ${ }^{16}$. También se eliminó del análisis la variante [t], ya que solo representa el $1 \%$ de las instancias de variación. De esta forma, la Tabla 2 muestra la distribución de ( ()) para las tres variantes restantes, para los hablantes no categóricos.

Tabla 2.

Distribución de (f) en las variantes [f], [t] y [t]], para hablantes no categóricos $(N=243)$.

\begin{tabular}{cccccccc}
\hline & \multicolumn{3}{c}{$[\mathrm{f}]$} & \multicolumn{3}{c}{$[\mathrm{f}]$} & \multicolumn{3}{c}{$[\mathrm{t}]$} \\
\cline { 2 - 8 } & $\%$ & $\mathrm{~N}$ & $\%$ & $\mathrm{~N}$ & $\%$ & $\mathrm{~N}$ \\
\hline Total & 67 & 162 & 24 & 57 & 10 & 24 \\
\hline
\end{tabular}

Fuente: Elaboración propia.

15 El valor sociolingüístico de este segmento es diferente en Chile y en Ciudad de Panamá, ya que mientras en Chile está estigmatizado, en Panamá es prestigioso.

${ }_{16}$ Véase también Smith, Durham y Fortune (2007) y Smith, Durham y Richards (2013) para una justificación similar en relación con la remoción de hablantes categóricos. 


\subsection{Análisis factor por factor}

\subsubsection{Factores sociales}

Como fue mencionado anteriormente, los factores sociales estudiados fueron: el efecto de los hablantes de forma individual, el sexo, los años de instrucción en inglés y el grupo socioeconómico de los hablantes. Sin embargo, el análisis preliminar de los resultados indicó que el único factor que parecía tener incidencia en la variación era el nivel socioeconómico ${ }^{17}$. En relación con la variante estándar, el comportamiento de los tres grupos difirió levemente el uno del otro, desde el grupo medio-bajo al medio-alto. La progresión en el uso de la forma meta no pareció ser reflejada por las variantes no meta, como se muestra en la siguiente tabla.

Tabla 3.

Distribución de la fricativa alveolar sorda (/) por grupo socioeconómico de los hablantes no categóricos $(N=243)$.

\begin{tabular}{lcccccc}
\hline & \multicolumn{2}{c}{ Fricativa } & \multicolumn{2}{c}{ Africada } & \multicolumn{2}{c}{ Fricativizada } \\
\hline & \multicolumn{2}{c}{$[\mathrm{f}]$} & & \multicolumn{2}{c}{$[\mathrm{t}]$} & \multicolumn{3}{c}{$[\mathrm{t}]$} & \\
\hline Grupo socioeconómico & $\%$ & $\mathrm{~N}$ & $\%$ & $\mathrm{~N}$ & $\%$ & $\mathrm{~N}$ \\
\hline Medio-medio & 76 & 44 & 19 & 11 & 5 & 3 \\
\hline Medio-alto & 68 & 47 & 22 & 15 & 10 & 7 \\
\hline Medio-bajo & 61 & 71 & 27 & 31 & 12 & 14 \\
\hline Total & 67 & 162 & 24 & 57 & 10 & 24 \\
\hline
\end{tabular}

Fuente: Elaboración propia.

El uso de africadas fue similar en los grupos medio-alto y mediobajo y la fricativizada fue usada exclusivamente por los grupos mediomedio y medio-bajo. La variación fue estadísticamente no significativa (valor $p=0,3721335 ; \chi^{2}=4,26$ ), por lo que no habría correlación en el uso de las variantes y los factores estudiados; sin embargo, el uso de estas tres variantes es interesante por las siguientes razones:

17 Del análisis individual de los hablantes se desprende que la mayoría utiliza la forma objeto por sobre las demás, con excepción de un solo hablante. El factor sexo no pudo ser analizado, porque tanto hombres como mujeres mostraron un comportamiento intragrupal disímil, al igual que el análisis del factor de años de experiencia en la L2. 
- La fricativa es estigmatizada en la L1 de los hablantes. El patrón sugiere que los hablantes de clase media-baja muestran el uso más bajo de esta variante $(61 \%, \mathrm{~N}=71)$, mientras que el uso más alto ocurre en el grupo medio-medio (76\%). En este caso, los hablantes de clase media-baja parecen evitar el uso de la variante estigmatizada.

- Las variantes no meta reflejan el patrón de la variante meta.

- En el lado opuesto de la fricativa, la forma prestigiosa en la Ll de este grupo de hablantes está africada. Esto se demuestra en el hecho de que el uso más alto de esta variante ocurre en el grupo medio-bajo (27\%) y el más bajo, en el grupo medio-medio (16\%). Tradicionalmente, el fenómeno de la hipercorrección ha sido vinculado con hablantes del grupo socioeconómico medio-bajo, tanto en situaciones de lengua materna (Labov, 1966, 1990) como en casos de segunda lengua (Beebe, 1980); en este estudio, dichos patrones parecen replicarse.

- Finalmente, en relación con los grupos socioeconómicos, el uso de la fricativizada sigue los mismos patrones que la africada (medio-medio > medio-alto > medio-bajo). Si lo consideráramos como un segmento en desarrollo, podríamos decir que sigue el mismo principio de la hipercorrección. En otras palabras, podríamos esperar que los hablantes que son conscientes del valor sociolingüístico de este sonido en la L1 - ya que su proceso de adquisición está aún incompleto-, podrían elegir la variante prestigiosa por sobre la estigmatizada, lo que explicaría el patrón que aquí se muestra.

Aunque los resultados que comparan estos tres grupos parecen contribuir evidentemente a la variación, las diferencias por grupo socioeconómico no son lo suficientemente robustas en sí mismas.

\subsubsection{Factores internos}

Dentro de los factores lingüísticos que se analizaron se cuentan: el segmento fonético anterior y posterior, la posición en la estructura prosódica (inicial y final) y el ítem léxico. Se espera que, como en otros estudios (Cedergren, 1973), el contexto fonético sea uno de los factores condicionantes más importantes. 


\subsubsection{1. Ítem léxico}

Solo se examinaron los ítems léxicos con más de 10 instancias de uso; la categoría "otros" incluye todos los ítems léxicos con menos de 10 apariciones.

Tabla 4.

Distribución de la fricativa alveolar sorda (/) por item léxico, en hablantes no categóricos $(N=243)$.

\begin{tabular}{|c|c|c|c|c|c|c|}
\hline & \multicolumn{2}{|c|}{ Fricativa } & \multicolumn{2}{|c|}{ Africada } & \multicolumn{2}{|c|}{ Fricativizada } \\
\hline \multirow[t]{2}{*}{ Ítem léxico } & \multicolumn{2}{|c|}{$\left[\int\right]$} & \multicolumn{2}{|c|}{ [tg] } & \multicolumn{2}{|c|}{$[\mathrm{t} f]$} \\
\hline & $\%$ & $\mathrm{~N}$ & $\%$ & $\mathrm{~N}$ & $\%$ & $\mathrm{~N}$ \\
\hline Show, shown, shows & 87 & 13 & 13 & 2 & 0 & 0 \\
\hline Short, shorter, shorts & 86 & 12 & 14 & 2 & 0 & 0 \\
\hline Relationship & 80 & 12 & 20 & 3 & 0 & 0 \\
\hline Finish, finishing & 71 & 10 & 14 & 2 & 14 & 2 \\
\hline English & 70 & 30 & 9 & 4 & 21 & 9 \\
\hline Should & 64 & 7 & 9 & 1 & 27 & 3 \\
\hline She & 61 & 43 & 37 & 26 & 3 & 2 \\
\hline Share, sharing & 60 & 6 & 30 & 3 & 10 & 1 \\
\hline Otros $(<10)$ & 60 & 12 & 35 & 7 & 5 & 1 \\
\hline Spanish & 57 & 17 & 23 & 7 & 20 & 6 \\
\hline Total & 67 & 162 & 24 & 57 & 10 & 24 \\
\hline
\end{tabular}

Fuente: Elaboración propia.

En primera instancia se examinó el condicionamiento de los ítems léxicos en términos de clase de palabra (función versus contenido), porque se determinó que los ítems léxicos de frecuencia alta de uso se comportaban de forma diferente de los de baja frecuencia (véase, por ejemplo, Bell et al., 2001) y, en segundo término, hay ciertas similitudes en la naturaleza de las palabras escogidas, por ejemplo, la posición de la variable, la clase, el contexto fonético en que ocurre la variable, etc. Se esperaba un mayor uso de formas débiles (fricativas, como resultado del proceso de lenición), en palabras de función que en palabras de contenido, las que se verían fortalecidas por efecto del componente oclusivo de las africadas. 
La mayoría de los ítems léxicos corresponde a palabras de contenido $^{18}$, con la excepción de should $(5 \%, \mathrm{~N}=11)$ y she $(29 \%, \mathrm{~N}=71)$. La variación de estos dos términos es similar en el segmento meta, pero no en las formas no meta. Should muestra una jerarquía de desarrollo, con un mayor uso de la fricativizada (similar al ítem English), mientras que en she, el uso de la africada es mayor que en los demás ítems léxicos. Should y she se comportan de forma similar a las palabras de contenido.

En resumen, la clase de las palabras parece tener un rol importante en la variación de forma similar a la posición de la variable en la palabra y otras características estructurales, como en los ítems English y Spanish, los que no solo presentan la variable en posición final, sino que también son sustantivos propios, con el mismo segmento anterior.

\subsubsection{Segmento fonético}

Históricamente, los estudios variacionistas de segmentos fonéticos han considerado la observación del segmento anterior y posterior como uno de los factores que más contribuyen a la variación (véase, por ejemplo, la contribución del contexto fonético en variables como la supresión de (t, d) en Bayley, 1994; Guy, 1994; Tagliamonte, 1998; Tagliamonte y Temple, 2005; Santa Ana, 1996; o la supresión de (s) en el español de Puerto Rico, Poplack, 1980).

En el caso de esta variable, Cedergren (1973) confirma la hipótesis de que la presencia de una vocal anterior favorece la lenición, es decir, las vocales como segmento anterior favorecerían el uso de las fricativas por sobre el de las africadas. En este estudio cada segmento fonético fue identificado, pero por motivos prácticos, fueron luego agrupados por articulación ${ }^{19}$.

\footnotetext{
18 La categoría Otros incorpora de forma exclusiva palabras de clase abierta.

19 Todos los segmentos fonéticos anteriores se agruparon en categorías mayores para facilitar el análisis. Esta decisión se basa en trabajos como los de Cedergren (1973), Guy (1991), Poplack, Tagliamonte \& Eze (2000), Poplack y Tagliamonte (2001), entre otros. Guy (2007) sugiere que la naturaleza de los objetivos de la investigación determinará cómo se considera (y agrupa) el contexto fonético, es decir, dependerá del nivel de análisis que el investigador desee realizar.
} 


\section{a) Segmento fonético anterior}

Como todas las categorías difieren entre sí, no es posible considerar categorías mayores (por ejemplo, consonantes versus vocales, como en Cedergren, 1973). La siguiente tabla muestra los resultados para segmento fonético anterior.

Tabla 5.

Distribución de la fricativa alveolar sorda (J) para segmento fonético anterior para los grupos: pausa, vocales y consonantes; para hablantes no categóricos $(N=243)$.

\begin{tabular}{|c|c|c|c|c|c|c|}
\hline & \multirow{2}{*}{\multicolumn{2}{|c|}{$\begin{array}{c}\text { Fricativa } \\
{[J]} \\
\end{array}$}} & \multirow{2}{*}{\multicolumn{2}{|c|}{$\begin{array}{c}\text { Africada } \\
{[\mathfrak{t}]} \\
\end{array}$}} & \multirow{2}{*}{\multicolumn{2}{|c|}{$\begin{array}{c}\text { Fricativizada } \\
{\left[{ }^{\mathrm{t}}\right]} \\
\end{array}$}} \\
\hline & & & & & & \\
\hline & $\%$ & $\mathrm{~N}$ & $\%$ & $\mathrm{~N}$ & $\%$ & $\mathrm{~N}$ \\
\hline Fricativas & 87 & 13 & 7 & 1 & 7 & 1 \\
\hline Laterales & 86 & 6 & 14 & 1 & 0 & 0 \\
\hline Pausa & 83 & 5 & 17 & 1 & 0 & 0 \\
\hline Nasales & 70 & 19 & 30 & 8 & 0 & 0 \\
\hline Vocales & 67 & 99 & 20 & 29 & 14 & 20 \\
\hline Vibrante & 67 & 6 & 33 & 3 & 0 & 0 \\
\hline Oclusivas & 45 & 14 & 45 & 14 & 10 & 3 \\
\hline Total & 67 & 162 & 24 & 57 & 10 & 24 \\
\hline
\end{tabular}

Fuente: Elaboración propia.

Las diferencias sonestadísticamente significativas $(p=0,000006393875$; $\left.\chi^{2}=46,21\right)$. En este caso, las vocales no parecen favorecer el uso de las fricativas más que las oclusivas (como en Cedergren, 1973); es más, English y Spanish, ambas con el mismo segmento anterior, presentan un comportamiento diferente.

Por otra parte, es interesante observar que las oclusivas muestran un uso mayor de africadas y fricativizadas ( $45 \%$ y $10 \%$, respectivamente), considerando que ambas tienen un componente oclusivo en su articulación si se compara con las fricativas.

\section{b) Segmento fonético posterior}

$\mathrm{Al}$ igual que con el segmento fonético anterior, los segmentos se agrupan en categorías mayores, por lo que se plantean las mismas hipótesis: las vocales favorecerían el uso de las fricativas, así como las oclu- 
sivas, el de las africadas y fricativizadas. La siguiente tabla muestra los resultados.

Tabla 6.

Distribución de la fricativa alveolar sorda (J) para segmento fonético posterior $(N=243)$.

\begin{tabular}{lccccccc}
\hline & \multicolumn{2}{c}{ Fricativa } & \multicolumn{2}{c}{ Africada } & \multicolumn{2}{c}{ Fricativizada } \\
\hline & \multicolumn{2}{c}{$[\mathrm{f}]$} & & \multicolumn{2}{c}{$[\mathrm{t}]$} & & \multicolumn{2}{c}{$[\mathrm{t}] \mathrm{l}$} \\
\hline Segmento & $\%$ & $\mathrm{~N}$ & $\%$ & $\mathrm{~N}$ & $\%$ & $\mathrm{~N}$ \\
\hline Nasales & 100 & 5 & 0 & 0 & 0 & 0 \\
\hline Aproximantes & 100 & 3 & 0 & 0 & 0 & 0 \\
\hline Africadas & 100 & 1 & 0 & 0 & 0 & 0 \\
\hline Vibrante & 100 & 1 & 0 & 0 & 0 & 0 \\
\hline Pausa & 70 & 9 & 8 & 1 & 23 & 3 \\
\hline Vocales & 67 & 122 & 28 & 51 & 6 & 10 \\
\hline Oclusivas & 61 & 14 & 17 & 4 & 22 & 5 \\
\hline Fricativas & 50 & 7 & 7 & 1 & 43 & 6 \\
\hline Total & 67 & 162 & 24 & 57 & 10 & 24 \\
\hline
\end{tabular}

Fuente: Elaboración propia.

Los resultados son estadísticamente significativos ( $p=0,001395195$; $\left.\chi^{2}=35,15\right)$. La hipótesis que plantea que las vocales favorecerían el uso de la fricativa se confirma, pero las consonantes no parecen restringir su uso, como argumenta Cedergren (1973), al menos con el segmento fonético posterior. Es más, la fricativa muestra el patrón opuesto en el segmento anterior (como segmento posterior, presenta el mismo porcentaje de uso para las variantes meta y no meta). Solo las vocales y las oclusivas tienen un comportamiento consistente, donde las primeras tienen porcentajes y jerarquías de uso similare $\mathrm{s}^{20} \mathrm{y}$ las segundas un porcentaje relativamente bajo de uso de variantes meta, en comparación con las no meta.

20 Una tabulación cruzada del segmento anterior y el posterior confirma que la posición intervocálica favorece el uso de las fricativas $(67 \%, \mathrm{~N}=60)$, lo que es consistente con los patrones descubiertos por Cedergren (1973). 


\subsubsection{Posición en la palabra}

Se ha determinado que la posición prosódica es un factor de importancia en el estudio del fortalecimiento consonántico, donde la posición inicial tiende a favorecer el fortalecimiento y la posición media, la fricativización, especialmente en posición intervocálica (Fougeron, 2001; Fougeron \& Keating, 1997; Pérez, 2001, 2007). La Tabla 7 muestra los resultados para las posiciones inicial, media y final.

Tabla 7.

Distribución de la fricativa alveolar sorda (/) por posición en la palabra $(N=243)$.

\begin{tabular}{|c|c|c|c|c|c|c|}
\hline & \multicolumn{2}{|c|}{ Fricativa } & \multicolumn{2}{|c|}{ Africada } & \multicolumn{2}{|c|}{ Fricativizada } \\
\hline & \multicolumn{2}{|c|}{ [J] } & \multicolumn{2}{|c|}{$[\mathrm{t}]$} & \multicolumn{2}{|c|}{$\left[\mathrm{t} \int\right]$} \\
\hline Posición & $\%$ & $\mathrm{~N}$ & $\%$ & $\mathrm{~N}$ & $\%$ & $\mathrm{~N}$ \\
\hline Media & 77 & 20 & 23 & 6 & 0 & 0 \\
\hline Inicial & 67 & 84 & 28 & 35 & 5 & 6 \\
\hline Final & 63 & 58 & 17 & 16 & 20 & 18 \\
\hline Total & 67 & 162 & 24 & 57 & 10 & 24 \\
\hline
\end{tabular}

Fuente: Elaboración propia.

El efecto de la posición prosódica, es significativa ( $p=0,001402926$; $\left.\chi^{2}=17,71\right)$. De igual forma, los resultados son consistentes con la literatura, ya que en posición inicial el porcentaje de africadas es mayor que en posición media ( $28 \%$, en negrita); el mayor porcentaje de fricativas ocurre en posición media (77\%, también en negrita). Resulta interesante ver que en posición final hay un uso importante de formas no meta. La articulación de africadas - y de consonantes, en generalen español, no es tan frecuente como en inglés (Alvar, 1980); incluso menos común es la articulación de este tipo de segmentos, ya que las africadas y las fricativizadas tienen un componente oclusivo y fricativo que rara vez ocurre en español. Esto significa que los sujetos no solo han adquirido la estructura silábica de la L2 (en que la articulación de consonantes es más frecuente en la coda), sino que están utilizando la forma prestigiosa y la fusionada (de desarrollo) más que la forma meta.

Por medio de la tabulación cruzada de los factores ítem léxico y posición prosódica se examinó la interacción de estos dos factores, como se aprecia en la Tabla 8. 
Tabla 8.

Tabulación cruzada de item léxico y posición inicial, media y final $(N=243)$.

\begin{tabular}{|c|c|c|c|c|}
\hline & Ítem léxico & Variante & $\mathrm{N}$ & $\%$ \\
\hline \multirow{24}{*}{$\begin{array}{l}\text { Posición } \\
\text { inicial }\end{array}$} & \multirow{4}{*}{$\begin{array}{l}\text { Show, shown, } \\
\text { shows }\end{array}$} & Fricativa & 13 & 87 \\
\hline & & Africada & 2 & 13 \\
\hline & & Fricativizada & 0 & 0 \\
\hline & & $\Sigma$ & 15 & \\
\hline & \multirow{4}{*}{$\begin{array}{l}\text { Short, shorter, } \\
\text { shorts }\end{array}$} & Fricativa & 12 & 86 \\
\hline & & Africada & 2 & 14 \\
\hline & & Fricativizada & 0 & 0 \\
\hline & & $\Sigma$ & 14 & \\
\hline & \multirow{4}{*}{ Otros } & Fricativa & 3 & 75 \\
\hline & & Africada & 1 & 25 \\
\hline & & Fricativizada & 0 & 0 \\
\hline & & $\Sigma$ & 4 & \\
\hline & \multirow{4}{*}{ Should } & Fricativa & 7 & 64 \\
\hline & & Africada & 1 & 9 \\
\hline & & Fricativizada & 3 & 27 \\
\hline & & $\Sigma$ & 11 & \\
\hline & \multirow{4}{*}{ She } & Fricativa & 43 & 61 \\
\hline & & Africada & 26 & 37 \\
\hline & & Fricativizada & 2 & 3 \\
\hline & & $\Sigma$ & 71 & \\
\hline & \multirow{4}{*}{$\begin{array}{l}\text { Share, } \\
\text { sharing }\end{array}$} & Fricativa & 6 & 60 \\
\hline & & Africada & 3 & 30 \\
\hline & & Fricativizada & 1 & 10 \\
\hline & & $\Sigma$ & 10 & \\
\hline \multirow{12}{*}{$\begin{array}{l}\text { Posición } \\
\text { media }\end{array}$} & \multirow{4}{*}{$\begin{array}{l}\text { Finished, } \\
\text { finishing }\end{array}$} & Fricativa & 4 & 100 \\
\hline & & Africada & 0 & 0 \\
\hline & & Fricativizada & 0 & 0 \\
\hline & & $\Sigma$ & 4 & \\
\hline & \multirow{4}{*}{ Relationship } & Fricativa & 12 & 80 \\
\hline & & Africada & 3 & 20 \\
\hline & & Fricativizada & 0 & 0 \\
\hline & & $\Sigma$ & 15 & \\
\hline & \multirow{4}{*}{ Otros } & Fricativa & 4 & 57 \\
\hline & & Africada & 3 & 43 \\
\hline & & Fricativizada & 0 & 0 \\
\hline & & $\Sigma$ & 7 & \\
\hline
\end{tabular}




\begin{tabular}{|c|c|c|c|c|}
\hline \multirow{16}{*}{ Posición final } & \multirow{4}{*}{ English } & Fricativa & 30 & 70 \\
\hline & & Africada & 4 & 9 \\
\hline & & Fricativizada & 9 & 21 \\
\hline & & $\Sigma$ & 43 & \\
\hline & \multirow{4}{*}{ Finish } & Fricativa & 6 & 60 \\
\hline & & Africada & 2 & 20 \\
\hline & & Fricativizada & 2 & 20 \\
\hline & & $\Sigma$ & 10 & \\
\hline & \multirow{4}{*}{ Spanish } & Fricativa & 17 & 57 \\
\hline & & Africada & 7 & 23 \\
\hline & & Fricativizada & 6 & 20 \\
\hline & & $\Sigma$ & 30 & \\
\hline & \multirow{4}{*}{ Otros } & Fricativa & 5 & 56 \\
\hline & & Africada & 3 & 33 \\
\hline & & Fricativizada & 1 & 11 \\
\hline & & $\Sigma$ & 9 & \\
\hline
\end{tabular}

Fuente: Elaboración propia.

El análisis de estos dos factores en interacción revela que:

- Todos los ítems léxicos favorecen el uso de la forma meta (fricativa). Las tres variantes aparecen en posición inicial y final; sin embargo, solo las fricativas y africadas lo hacen en posición media.

- La frecuencia de uso de la forma meta disminuye en un patrón fricativa $>$ africada $>$ fricativizada, para la mayoría de los ítems léxicos, con la excepción de should, que lo hace en el patrón fricativa $>$ fricativizada $>$ africada $^{21 .}$

- En oposición a lo anterior, el uso de las formas no meta aumenta en posición media y final; el uso de africadas es mayor para la mayoría de los ítems léxicos, con la excepción de English, que muestra el mayor uso de la fricativizada, en posición final.

En resumen, los resultados indican que el uso de la forma meta es mayor en posición inicial y media, en tanto las formas no meta lo hacen en posición final. Esto es consistente parcialmente con la literatura

${ }_{21} \quad$ El patrón de variación de should es similar al de English. La variación relativamente alta de estas dos palabras resulta particularmente interesante dada la saliencia de estas palabras para los aprendices de inglés (Corpus of Contemporary American English (Davies, 2008) http://www.wordfrequency.info). 
que plantea que las articulaciones más fuertes ocurren en posición inicial (en este caso las africadas, por efecto de su componente oclusivo), mientras que la posición media tiende a favorecer la fricativización. El alto porcentaje de formas no meta en posición final sugiere que los sujetos han adquirido la estructura silábica del inglés, pero estarían aún en proceso de separar las características sociolingüísticas de esta variable de la L1 y la L2.

\subsubsection{Resumen de resultados}

\subsubsection{Resultados para los factores sociales}

Los resultados determinan la existencia de cuatro variantes para ( $($ ), lo que significa que la variación ocurre en la L2 de la misma forma que en español como L1; la variante más frecuente es la fricativa postalveolar sorda [ [], que corresponde a la forma meta del inglés, como fue hipotetizado. El análisis de factores determinó que todos los hablantes muestran preferencia por la forma meta, con excepción de un hablante. La significancia estadística de este grupo no se analizó debido a que los individuos no pueden ser analizados en relación con otros individuos, sino a partir de las características estudiadas como factores externos (sexo, tiempo de instrucción formal, grupo socioeconómico, etc.), al menos con el modelo de regresión utilizado en esta investigación (Johnson, 2009). La diferencia entre hombres y mujeres fue estadísticamente no significante, en tanto que la variación dentro de los dos grupos no fue homogénea, por lo que los patrones evidenciados en estudios similares (Major, 2004) no se replicaron para este segmento. Los resultados sugieren que la adquisición de la forma meta ocurre de manera gradual a medida que los sujetos progresan en el proceso adquisitivo, donde la mayoría de ellos la alcanza de modo casi categórico al final del quinto año de estudios. Aparentemente, se evidencia un patrón de desarrollo en el uso de las formas no meta. Sin embargo, las diferencias en la variación no son estadísticamente significantes y el análisis de variación intragrupal determinó un comportamiento heterogéneo. A pesar de que los resultados no son estadísticamente significativos, aquellos para el factor grupo socioeconómico son interesantes, ya que muestran que el patrón de variación de los tres grupos es homogéneo y con una aparente distinción en el uso de las variantes para cada uno de los grupos, especialmente en lo que respecta al mayor uso de la variante prestigiosa, en oposición a la 
variante meta en los hablantes del grupo medio-bajo, lo que resultaría en un caso de hipercorrección.

\subsubsection{Resultados para los factores lingüisticos}

En relación con el grupo ítem léxico, una vez que se separaron los sujetos categóricos de los no categóricos, se analizaron los ítems léxicos show (shown, shows), short (shorter, shorts), relationship, finish (finishing), English, should, she, share (sharing) y Spanish, más un grupo que incorporó todos los ítems con menos de 10 ocurrencias. El único patrón que surgió de este grupo es que se utilizaron las fricativas más que las otras dos variantes. El contexto fonético está asociado al ítem léxico, por lo que se realizó el análisis de estos para confirmar dicho fenómeno. Los resultados fueron significativos estadísticamente para los segmentos anterior y posterior; sin embargo, la contribución de este factor no fue tan marcada como lo sugiere la literatura. Es más, el efecto del segmento no parece estar asociado a los ítems léxicos con características estructurales similares, como sucede con las palabras English y Español. En términos generales, la posición en la palabra parece ser el principal factor que contribuye a la variación de $(\delta)$, dado que el uso de las fricativas y las africadas muestran una diferencia mucho más marcada que en los otros factores, en posición media e inicial, respectivamente. Este factor es estadísticamente significativo, en tanto que la interacción de la posición y el ítem léxico parece confirmar que el efecto de la posición, tal como del segmento anterior y posterior, dependen del ítem léxico.

Para comprobar la interacción de los factores, se realizó un análisis de variable múltiple.

\subsubsection{Análisis de variable múltiple}

Para realizar el análisis de variable múltiple, se analizó la aplicación de la regla solo en la variante meta [S] para evitar la ocurrencia de células nulas. Se eliminaron también del análisis del segmento fonético posterior todos los resultados categóricos, ya que no mostraban variación, dejando un total de 231 elementos de análisis. Los resultados se describen en la Tabla 9, donde se presentan de forma descendente los factores que más contribuyen a la variación. Se analizaron solo seis factores: grupo socioeconómico, tiempo de instrucción, ítem léxico, segmento anterior, segmento posterior y posición. 
Tabla 9.

Análisis de variable múltiple para la fricativa postalveolar sorda (()).

\begin{tabular}{|c|c|c|c|}
\hline & $\begin{array}{l}\text { Peso factorial } \\
\text { (FW) }\end{array}$ & $\%$ & Total N \\
\hline Input .654 & & 65 & 231 \\
\hline \multicolumn{4}{|c|}{ Contexto fonético anterior } \\
\hline Fricativas & ,77 & 87 & 15 \\
\hline Laterales & ,75 & 86 & 7 \\
\hline Pausa & ,72 & 83 & 6 \\
\hline Nasales & ,55 & 70 & 27 \\
\hline Vibrante &, 50 & 67 & 9 \\
\hline Vocales & ,49 & 65 & 137 \\
\hline Oclusivas & ,28 & 43 & 30 \\
\hline Rango & 49 & & \\
\hline \multicolumn{4}{|c|}{ Años de educación en inglés } \\
\hline Quinto año &, 71 & 83 & 41 \\
\hline Tercer año &, 50 & 66 & 84 \\
\hline Primer año & ,42 & 59 & 106 \\
\hline Rango & 29 & & \\
\hline \multicolumn{4}{|l|}{ Grupo socioeconómico } \\
\hline Medio-medio & ,61 & 75 & 56 \\
\hline Medio alto &, 51 & 67 & 63 \\
\hline Medio bajo & ,44 & 60 & 112 \\
\hline Rango & 17 & & \\
\hline \multicolumn{4}{|c|}{ Contexto fonético posterior } \\
\hline Pausa & {$[, 54]$} & 69 & 13 \\
\hline Vocales & {$[, 51]$} & 67 & 183 \\
\hline Pausa & {$[, 45]$} & 61 & 23 \\
\hline Fricativas & {$[, 35]$} & 50 & 12 \\
\hline \multicolumn{4}{|l|}{ Ítem léxico } \\
\hline Show, shown, shows & {$[, 77]$} & 87 & 15 \\
\hline Short, shorter, shorts & {$[, 75]$} & 86 & 14 \\
\hline Relationship & {$[, 67]$} & 80 & 15 \\
\hline English & {$[, 52]$} & 68 & 41 \\
\hline Should & {$[, 47]$} & 64 & 11 \\
\hline She & {$[, 43]$} & 60 & 70 \\
\hline Finish, finishing & {$[, 43]$} & 60 & 10 \\
\hline Otros & {$[, 41]$} & 59 & 29 \\
\hline Spanish & {$[, 37]$} & 54 & 26 \\
\hline \multicolumn{4}{|l|}{ Posición } \\
\hline Inicial & {$[, 51]$} & 67 & 123 \\
\hline Final & {$[, 44]$} & 60 & 82 \\
\hline Media & {$[, 25]$} & 77 & 26 \\
\hline
\end{tabular}

Fuente: Elaboración propia 
Como se observa, la interacción de solo tres grupos contribuye a la variación de (J): segmento fonético anterior (rango 49), tiempo de instrucción (rango 29) y grupo socioeconómico (rango 17). Se determina así que los otros tres factores, en interacción, no contribuyen a la variación.

\section{Resumen y conclusiones}

La hipótesis inicial que proponía que el uso de la fricativa postalveolar sorda $(\delta)$ sería replicado desde el español de Chile al inglés se refuta: los sujetos utilizan la forma meta [J] mucho más que la forma prestigiosa de la Ll [tg $]$, a pesar de que esta aparece fuertemente estigmatizada. Esto sugiere que los sujetos superan el paradigma social del segmento y reconocen las diferencias fonéticas entre la Ll y la L2. Sin embargo, el uso de las formas no meta parece estar motivado sociolingüísticamente, ya que se determina que tanto los factores internos como externos contribuyen en la variación de la fricativa alveolar sorda. Específicamente, el análisis de variable múltiple sugiere lo siguiente:

- El factor más importante es el segmento anterior (rango 49). En oposición a los resultados mostrados por Cedergren (1973), las vocales no parecen desfavorecer el uso de las fricativas a través del proceso de lenición. De este grupo de factores, las fricativas son las que promueven, en mayor medida, el uso de la variante fricativa $(, 77)$ y las oclusivas, las que menos lo hacen $(, 28)$. Así, este es el único factor interno que contribuye a la variación de ( () ).

- El segundo factor significativo es el tiempo de instrucción (rango 29), que demuestra una progresión clara en la adquisición de la forma meta desde el primer año $(, 42)$, seguido por el tercero $(, 50)$ y, finalmente, el quinto año (,71).

- Finalmente, el tercer factor que contribuye a la variación es el grupo socioeconómico (rango 17). Los resultados confirman lo sugerido por Labov $(1966,1990)$ y Beebe (1980), que plantean que la hipercorrección ocurre en sujetos del grupo medio-bajo. Aquí, este grupo muestra un uso relativamente alto de las formas no meta, que son más prestigiosas, comparado con la forma meta $(, 44)$. El mayor uso de la forma meta ocurre en los sujetos del grupo medio-medio. 
De las hipótesis probadas, el efecto del tiempo de instrucción es uno de los más relevantes en el estudio de la variación en la adquisición de una segunda lengua, ya que muestra que la adquisición de formas meta está conectada con el tiempo de exposición a la L2, aun en contextos de lengua extranjera.

\section{Referencias bibliográficas}

Alvar, A. M. (1980). Los fonemas implosivos en español. Thesaurus, 35(3), 456-505. Recuperado de https://cvc.cervantes.es/lengua/thesaurus/pdf/35/TH_35_003_012_0.pdf

Bayley, R. (1994) Interlanguage variation in the quantitative paradigm: Past-Tense marking in Chinese-English. In: E. Tarone, S.M, Gass, and A. Cohen (eds.), Research methodology in second language acquisition. (pp. 157-181). Hillsdale, NJ: Lawrence Erlbaum.

Beebe, L. M. (1980). Sociolinguistic variation and style shifting in second language acquisition. Language Learning, 30(2), 433445. https://doi.org/10.1111/j.1467-1770.1980.tb00327.x

Bell, A., Jurafsky, D., Fosler-Lussier, E., Girand, C., Gregory, M., and Gildea, D. (2001) Form variation of English function words in conversation. Submitted manuscript.

Bernales, M. (1978). Sobre la palatal africada en el español de la ciudad de Valdivia. RLA: Revista de Lingüistica Teórica y Aplicada, $16,41-52$.

Canfield, D. L. (1962). La pronunciación del español de América. Bogotá: Instituto Caro y Cuervo.

Catford, J. C. (2002). A practical introduction to phonetics (second edition). Oxford: Oxford University Press.

Cedergren, H. (1973). The interplay of social and linguistic factors in Panama (PhD dissertation). Cornell University, Ithaca.

Cedergren, H. (1987). The spread of language change: Verifying inferences of linguistic diffusion. En P. H. Lowenberg (Ed.), Language spread and language policy: Issues, implications and case studies (pp. 45-60). Washington D.C: Georgetown University Press.

Cepeda, G. (2001). Estudio descriptivo del español de Valdivia, Chile. Estudios Filológicos 36, 81-97. https://doi.org/10.4067/ s0071-17132001003600006 
Chambers, J. K. (1980). Linguistic variation and Chomsky's homogenous speech community. En M. Kinloch \& A. B. House (Eds.), Papers from the fourth Annual Meeting of the Atlantic Provinces Linguistics Association (pp. 1-32). Fredericton: University of New Brunswick.

Chambers, J. K. \& Trudgill, P. (1998). Dialectology. Cambridge: Cambridge University Press.

Dalbor, J. B. (1959). The English phonemes /š/ and /č/: A hearing and pronunciation problem for speakers of Spanish learning English. Language Learning, 9(1-2), 67-73. https://doi. org/10.1111/j.1467-1770.1959.tb01131.x

Davies, M. (2008). The corpus of contemporary American English. BYE, Brigham Young University.

Eklund, R. \& Lindström, A. (1998). How to handle "foreign" sounds in Swedish text-to-speech conversion: Approaching the 'xenophone' problem. Recuperado de https://www.ida.liu. se/ robek28/pdf/Eklund_Lindstrom_1998_Xenophones. pdf

Flege, J. E. (1991). Age of learning affects the authenticity of voiceonset time (VOT) in stop consonants produced in a second language. Journal of the Acoustical Society of America, 89(1), 395-411. https://doi.org/10.1121/1.400473

Fougeron, C. (2001). Articulatory properties of initial segments in several prosodic constituents in French. Journal of Phonetics, 29(2), 109-135. https://doi.org/10.1006/jpho.2000.0114

Fougeron, C. \& Keating, P. A. (1997). Articulatory strengthening at edges of prosodic domains. The Journal of the Acoustical Society of America, 101(6), 3728-3740. https://doi. org/10.1121/1.418332

Guy, G. R. (1991). Explanation in variable phonology. An exponential model of morphological constraints. Language Variation and Change, 3(1), 1-22. https://doi.org/10.1017/ s0954394500000429

Guy, G. R. (1994) Variable is variable: Principles, constraints, and linguistic variation. Paper presented at NWAVE-XXIII, Stanford University,

Guy, G. R. (2007). Lexical exceptions in variable phonology. University of Pennsylvania Working Papers in Linguistics, 13(2), 108- 
119. Recuperado de https://repository.upenn.edu/cgi/viewcontent.cgi?article $=1007 \&$ context $=$ pwpl

Johnson, D. E. (2009). Getting off the GoldVarb standard: Introducing Rbrul for Mixed-Effects Variable Rule Analysis. Language and Linguistics Compass, 3(1), 359-383. https://doi. org/10.1111/j.1749-818x.2008.00108.x

Jongman, A., Wayland, R., \& Wong, S. (2000). Acoustic characteristics of English fricatives. Journal of the Acoustical Society of America, 108(3), 1252-1263. https://doi.org/10.1121/1.1288413

Kpogo, F. \& Gathercole, V. C. M. (2019). The influence of native English-speaking environment on Akan-English bilingual' production of English inter-dental fricatives. International Journal of Bilingualism, 1367006919844032. https://doi. org/10.1177/1367006919844032

Labov, W. (1966). The social stratification of English in New York City. Washington, D.C.: Center for Applied Linguistics.

Labov, W. (1972a) Sociolinguistic Patterns. Philadelphia: University of Pennsylvania Press.

Labov, William. (1984). Field methods of the Project on Linguistic Change and Variation. In Baugh, J. \& Sherzer, J. (eds.), Language in Use. Englewood Cliffs: Prentice Hall. 28-53.

Labov, W. (1990). The intersection of sex and social class in the course of linguistic change. Language Variation and Change, 2(2), 205-54. https://doi.org/10.1017/s0954394500000338

Labov, W. (2001). Principles of linguistic change, Vol. II: Social factors. Oxford: Blackwell.

Labov, W. (marzo, 2012). The sociolinguistic intersection of Spanish and English. En J. E. Alatis, TESOL Convention, Philadelphia.

Ladefoged, P. \& Disner, S. F. (2012). Vowels and consonants. New Jersey: John Wiley \& Sons.

Ladefoged, P. \& Johnstone, K. (2011). A course in phonetics (sixth edition). Boston: Wadsworth Cengage Learning.

Levon, E. (2007). Sexuality in context: Variation and the sociolinguistic perception of identity. Language in Society, 36(04), 533-554. https://doi.org/10.1017/s0047404507070431

Li, F., Edwards, J., \& Beckman, M. E. (2009). Contrast and covert contrast: The phonetic development of voiceless sibilant frica- 
tives in English and Japanese toddlers. Journal of Phonetics, 37(1), 111-124. https://doi.org/10.1016/j.wocn.2008.10.001

Lipski, J. (1994). Latin American Spanish. Nueva York: Longman.

MacWhinney, B. (2000). The CHILDES Project: Tools for analyzing talk. transcription format and programs (Vol. 1). Psychology Press.

Major, R. C. (2004). Gender and stylistic variation in second language phonology. Language Variation and Change, 16, 169-188. https://doi.org/10.1017/s0954394504163059

Miatto, V., Hamann, S., \& Boersma, P. (2019). Self-reported L2 input predicts phonetic variation in the adaptation of English final consonants into Italian. Canberra: Australasian Speech Science and Technology Association Inc.

Pérez, H. E. (2001). La noción de rasgo. El caso de las consonantes oclusivas el español. Onomázein, 6, 327-336. Recuperado de http://www2.udec.cl/ heperez/oldpage/rasgo.htm

Pérez, H.E. (2007). Estudio de la variación estilística de la serie /bdg/ en posición intervocálica en el habla de los noticieros de la televisión chilena. Estudios de Fonética Experimental, 16, 228259. Recuperado de https://repositorio.uc.cl/bitstream/handle/11534/7947/000496876.pdf?sequence=1\&isAllowed=y

Poplack, S. (1980) Sometimes I'll start a sentence in English y termino en Español: toward a typology of code-switching. Linguistics 18, pp. 581-618.

Poplack, S. \& Tagliamonte, S. (2001). African American English in the Diaspora. Oxford: Blackwell.

Poplack, S., Tagliamonte, S., \& Eze, E. (2000). Reconstructing the source of early African American English plural marking: A comparative study of English and Creole. En S. Poplack (Ed.), The English history of African American English (pp. 73-105). Oxford y Malden: Blackwell.

Santa Ana, O. (1996) Sonority and syllable structure in Chicano English. Language Variation and Change 8, pp. 3-91.

Sapir, E. (1921). Language: An introduction to the study of speech. New York: Harcourt, Brace and Company.

Shadle, C., Proctor, M. I., \& Iskarous, K. (2008). An MRI study of the effect of vowel context on English fricatives. Journal of the Acoustical Society of America, 123(5), 3735-3735. https:// doi.org/10.1121/1.2935246 
Smith, J. (2007). Methods in sociolinguistic research: syntax and morphology. En C. Llamas, L. Mullany, \& P. Stockwell (Eds.), The Routledge companion to sociolinguistics. London: Routledge.

Smith, J., Durham, M., and Fortune, L. (2007) "Mam, my trousers is fa'in doon!": Community, caregiver, and child in the acquisition of variation in a Scottish dialect. Language Variation and Change, 19(01), pp. 63-99.

Smith, J., Durham, M., and Richards, H. (2013) The social and linguistic in the acquisition of sociolinguistic norms: caregivers, children, and variation. Linguistics, 51(2), pp. 285-324.

Stuart-Smith, J., Timmins, C., \& Wrench, A. (2003). Sex and gender in /s/ in Glaswegian. En M. J. Solé, D. Recasens, \& J. Romero (Eds.), Proceedings of the 15th International Congress of Phonetic Sciences: 3-9 August 2003, Barcelona, Spain (pp. 1851-1854). Rundle Mall: Causal Productions.

Stuart-Smith, J. (2007). The influence of the media. En C. Llamas, L. Mullany \& P. Stockwell (Eds.), The Routledge companion to sociolinguistics (pp. 140-148). London: Routledge.

Subiabre Ubilla, P. B. (2015). A phonetic variationist study on Chilean speakers of English as a foreign language (Doctoral dissertation). University of Glasgow, Glasgow.

Tagliamonte, S. (1998) Was/were across the generations: view from the city of York. Language variation and change, 10 (2), pp. 153-92.

Tagliamonte, S. (2006). Analysing sociolinguistic variation. Cambridge: Cambridge University Press.

Tagliamonte, S. and Temple, R. (2005) New perspectives on an ol' variable: $(\mathrm{t}, \mathrm{d})$ in British English. Language Variation and Change, 17, pp. 281-302

Tassara, G. (1992). Actitudes lingüísticas ante la variación de [č]. $R L A$ : Revista de Lingüística Teórica y Aplicada, 30, 263-271.

Valencia, A. (1993-1994) Realizaciones de Išl, [č]/ y [ĵl en el habla adolescente. Nueva Revista del Pacifico, 38/39, pp. 159-180.

Valdivieso, H. (1983). Prestigio y estigmatización. Factor determinante en la enseñanza institucionalizada de la lengua materna. RLA: Revista de Lingüistica Teórica y Aplicada, 21, 137-142.

Valdivieso, H. (1993). Perfil fonético de escolares de Concepción. RLA: Revista de Lingüística Teórica y Aplicada, 31, 119-135. 
Valencia, A. (1993-1994). Realizaciones de Išl, [č] y [ĵl] en el habla adolescente. Nueva Revista del Pacífico, 38/39, 159-180.

Weinreich, U., Labov, W., \& Herzog M. I. (1968). Empirical foundations for a theory of language change. En W. P. Lehmann \& Y. Malkie (Eds.), Directions for historical linguistics: A symposium (pp. 95-188). Austin: University of Texas Press.

Wigdorsky, L. (1978). Realización de algunos fonemas consonánticos en el castellano de Santiago: informe preliminar. RLA: Revista de Lingüística Teórica y Aplicada, 16, 53-60.

Wilde, L. F. (1995). Analysis and synthesis of fricative consonants (Doctoral dissertation). Massachusetts Institute of Technology, Massachusetts. 